\section{The Effects of Combining High-Top Shoes with Twister Wrap Orthoses on Balance Parameters of Children with Spastic Diplegic Cerebral Palsy}

\author{
Mehrdad Davoudi' ${ }^{1 \oplus}$, Mobina Khosravi Farsani², Taher Ba- \\ baee ${ }^{3}$, Hamideh Ranjbar ${ }^{4}$, Seyyed Mohammadreza Shok- \\ ouhyan $^{5}$, Alireza Ghaznavi ${ }^{6}$, Mehdi Rezaei ${ }^{7 * \odot}$
}

\begin{abstract}
Background: Cerebral palsy $(\mathrm{CP})$ is a non-progressive encephalopathy before, during, or after childbirth with almost the most common type, i.e. spastic diplegic, leading to a frequent walking problem, In-toeing. Orthoses can reduce the consequences of CP.

Objective: This study aimed to evaluate the effect of combining twister wrap orthoses (TWO) with high-top shoes on the balance parameters of children with spastic diplegic $\mathrm{CP}$.

Material and Methods: In this quasi-experimental study, twenty children (aged $6.8 \pm 0.5$ years) with spastic diplegic $\mathrm{CP}$ with in-toeing gait participated. The tests were conducted in three conditions: 1) in bare-foot, 2) with high-top shoes, and 3 ) with high-top shoes plus TWO and the orthoses effects on balance parameters were compared.
\end{abstract}

Results: High-top shoes positively decreased center of pressure (COP) sway in the anterior-posterior (AP) direction. Both high-top shoes and high-top shoes with TWO conditions compared to bare-foot conditions significantly improved standing balance by decreasing the ellipse area. Pairwise, wearing TWO and high-top shoes significantly reduced the COP sway in the medial-lateral (ML) and AP directions with a significant difference between using the combined orthoses and the shoe without TWO in ML of COP displacement.

Conclusion: High-top shoes alone and the combination of high-top shoes with TWO conditions may enhance the stability of children with spastic diplegia more than barefoot. Although the use of combined orthoses induced significant improvement in the ML direction of COP displacement.

Citation: Davoudi M, Khosravi Farsani M, Babae T, Ranjbar H, Shokouhyan SM, Ghaznavi A, Rezaei M. The Effects of Combining High-Top Shoes with Twister Wrap Orthoses on Balance Parameters of Children with Spastic Diplegic Cerebral Palsy. J Biomed Phys Eng. 2022;12(1):91100. doi: 10.31661/jbpe.v0i0.2106-1358.

\section{Keywords}

Spastic Diplegic; Cerebral Palsy; Orthoses; Twister Wrap Orthoses; Balance; In-toeing; Walking; Shoes

\section{Introduction}

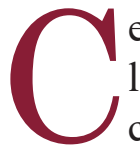
erebral palsy $(\mathrm{CP})$ is a type of upper motor neuron disturbance, leading to abnormal muscle tone and decreasing selective motor control of the limbs [1]. CP varies based on prenatal care and socio-economic status, type of delivery, and postpartum care ranging from two to four patients per 1000 live births [2]. The spastic diplegic
${ }^{1} \mathrm{MSc}$, Department of Mechanical Engineering, Sharif University of Technology, Tehran, Iran

${ }^{2} \mathrm{MSc}$, Rehabilitation Research Center, Department of Orthotics and Prosthetics, School of Rehabilitation Sciences, Iran University of Medical Sciences, Tehran, Iran

${ }^{3} \mathrm{PhD}$, Rehabilitation Research Center, Department of Orthotics and Prosthetics, School of Rehabilitation Sciences, Iran University of Medical Sciences, Tehran, Iran

${ }^{4} \mathrm{MSc}$, Physiotherapy Research Center, School of Rehabilitation, Babol University of Medical Sciences, Babol, Iran

${ }^{5} \mathrm{MSc}$, Department of Mechanical Engineering, Sharif University of Technology, Tehran, Iran

${ }^{6} \mathrm{MD}$, Department of Orthopedic Surgery, Iran University of Medical Sciences, Tehran, Iran

${ }^{7} \mathrm{PhD}$, School of Rehabilitation, Shahid Beheshti University of Medical Sciences, Tehran, Iran

*Corresponding author: Mehdi Rezaei

School of Rehabilitation, Shahid Beheshti University of Medical Sciences Tehran, Iran

E-mail: rezaei.to@gmail. com

Received: 21 June 2021 Accepted: 27 September 2021 
is the most common type of CP in $32 \%$ of all children with $\mathrm{CP}$ [3], resulting in muscle resistance against movement $[4,5]$.

In spastic diplegic $\mathrm{CP}$, motor deficits and lower limbs' spasticity affect walking ability, causing a walking pattern determined by equinus ankle, exorbitant knee flexion, genu valgus, increased hip adduction, and internal rotation [6]. Moreover, in spastic diplegic, excessive femoral anteversion and tibial rotation significantly affect the soleus and gluteus medius muscles. Thus, the lower extremity on the affected side rotates internally, creating in-toeing movement. However, poor balance control is the result of poor walking abilities [7], in-toeing movement causes walking abnormality and disturbances in balance along with coordination [6-8]. Therefore, providing stability during dynamic movements and static positions is inevitable due to inherently poor balance in spastic diplegic with in-toeing [9].

Occupational therapy, physical therapy, and orthoses are the most common non-surgical treatments for children with mild to moderate deformities [9]. High-top shoes and ankle-foot orthosis are frequently prescribed to enhance ankle joint stability in CP patients $[10,11]$. Using twister cables or twister wrap orthosis (TWO) attached to waistbands proximally and the feet distally [12] aims to control the abnormal femoral/tibial rotations with in-toeing in children with spastic diplegic CP $[13,14]$. Although both TWO and twister cables have a similar functional mechanism, TWO is more convenient [14] with better effectiveness on gait parameters than twister cable [14].

Few studies are conducted about evaluating TWO on CP [13, 14]. According to the results [15], orthoses with the thigh segment of an elastic band wrapped around the limb lead to active rotational forces and changes in the foot progression angle.

Based on the study of Eid et al. that is the first and only study in this field and assessed the effect of combining TWO with ankle-foot orthoses in children with spastic diplegic $\mathrm{CP}$, the participants had a better foot pressure distribution and balance control using TWO with ankle-foot orthoses than ankle-foot orthosis alone [16]. Despite the effectiveness of high-top shoes in reducing the consequences of CP [17] and more convenience and costeffectiveness of high-top shoes compared to ankle-foot orthoses, the effects of TWO and high-top shoes on postural balance in children with CP is still unclear. Therefore, the present study aimed to evaluate the combined effect of TWO with high-top shoes on balance parameters of CP children. We hypothesized that TWO through creating out-toeing moments can increase the patient's base of support and improve balance. Thus, using TWO for increasing balance in the ML direction might be reasonable.

\section{Material and Methods}

\section{Subjects}

In this quasi-experimental study, twenty spastic diplegic CP children (aged $6.8 \pm 0.5$ years old) with in-toeing gait were referred by an orthopedic surgeon to a biomechanics laboratory of Shahid Beheshti University. The inclusion criteria were: 1) ages ranging from six to eight years old, 2) spastic diplegic with in-toeing gait, 3) Gross Motor Function Classification System (GMFCS) criteria of level 1, and 4) walking ability independently, but with problems in velocity, balance, and coordination [18]. The exclusion criteria consist of as follows: 1) a history of orthopedic surgery or botulinum toxin injection up to six months before the study [19], 2) lower limb fracture along with subluxation or dislocation of the lower limb joints [20], and 3) difficulty in hearing or vision [13]. Before recruiting, the purpose and study procedure were explained to the parents of all children, and a written informed consent form was signed before doing the tests on their children. The Ethics Committee also approved the study protocol (IR. SBMU.RETECH.REC.1399.372). 
Sampling size

The sample size was calculated by $\mathrm{G}$ power software considering power $80 \%$ and Alfa 0.05 [16].

\section{Interventions \\ High-top shoes}

The shoes' characteristics consist of 1) a sufficient width to reduce pressure on the medial and lateral sides of the feet, 2) reinforced medial counter to enhance rearfoot control, 3) shock absorber insoles, and 4) a high toe box with forefoot flexion and extension. Accommodative insoles were placed in the shoes to distribute the foot pressure evenly. All the high-top shoes were designed and fabricated for each patient individually and an expert orthotist approved their appropriateness.

\section{Twister wrap orthoses}

A TWO was custom-made for each child based on his/her size and an expert orthotist was responsible for donning the TWO for each child, while the child stood upright. Then the elastic strap was wrapped twice around his/ her lower leg continuously in the lateral direction (once below the knee, and then above it) and affixed to a thermoplastic pelvic belt that covered the pelvis at the proximal [21]. TWO was easily attached to the shoes (Figure 1). A clinical criterion was used to determine the amount of the strap strain, adjusted when the child's feet were positioned in a 5-degree toeout [22]. To set the child's feet in the proper toe-out angle, a two-arm goniometer was utilized. The long axis of the foot (from mid-heel to the second toe) was marked with a red line on each shoe. The strain amount of the strap was tolerable for all participants.

\section{Data collection}

All tests were conducted at the biomechanics laboratory of Shahid Beheshti University, while each child stood on a force platform (Bertec, Columbus, Ohio, USA) for the 20s (frequency of $100 \mathrm{~Hz}$ ) [23]. The data extracted from the force plate were the center of pres-

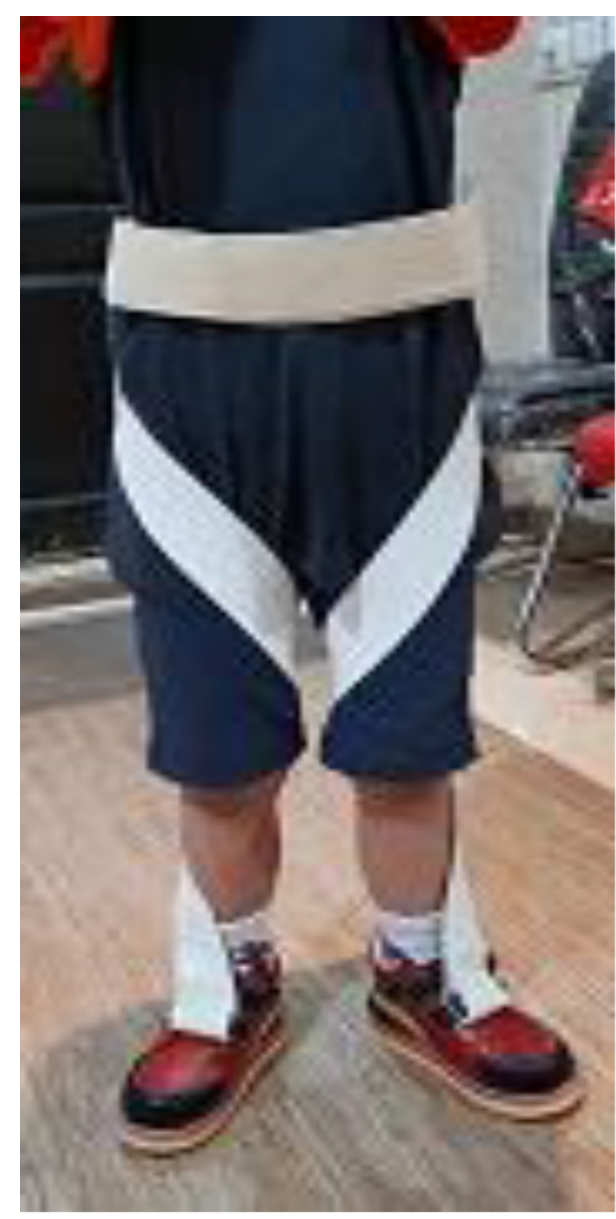

Figure 1: Twister Wrap Orthosis

sure positions in $\mathrm{x}$ (anterior-posterior (AP)) and y (medial-lateral (ML)) axes. Three different test conditions were recorded for each child as follows: 1) the child stood bare-foot, 2) the examiner put the child on the high-top shoes (both feet) while standing on the force plate, and 3) the shoes and the TWO were applied together and the test was then conducted. Moreover, each test was done three times with a two-minute rest interval between the repeats $[21,24,25]$ and each child wore an orthosis for 15 mins and walked in a laboratory environment before each test condition to accommodate the interventions.

\section{Data analysis}

A low-pass barter filter was applied to the data with a cut-off frequency of $5 \mathrm{~Hz}$ [26] 
in MATLAB software (Math Works, Natick, MA, USA). The main outcome was balance analysis with the following parameters: the standard deviation of displacement and velocity of the center of pressure for both the AP and the ML directions, the mean velocity of the center of pressure, the total path length of the center of pressure displacement, and the 95\% confidence ellipse area. An ellipse was considered as a plane curve surrounding two focal points; therefore, the sum of the two distances from the focal points is constant for all points on the curve [23]. The ellipse area explains fluctuations of the center of pressure and the changes in phase plane portrait for AP and ML directions. In addition, the total (overall) phase was calculated [24] (Table 1).

\section{Statistical analysis}

All the statistical analyses were done using SPSS software version 20.0, data normality was checked using the Kolmogorov-Smirnov test, and data were reported as mean and standard deviation. For comparing between the three conditions: 1) in barefoot (without intervention), 2) with high-top shoes, 3) with the high-top shoes and twister wrap orthoses, all the mentioned parameters (section 2.4) were compared with repeated measure one-way analysis of variance (ANOVA) and Bonferroni post hoc test. Pairwise comparisons were done to examine the main effects from repeated measure ANOVA with the level of significance was 0.05 .

\section{Results}

Twenty children with spastic diplegic CP with in-toeing participated in the present study (14 boys, six girls, aged $6.8 \pm 0.5$ years old). According to the Kolmogorov-Smirnov test, all data had a normal distribution. Tables 2 and 3 show the effect of conditions on the dependent linear variables. In addition, the pairwise comparisons (Table 4) show the results of the Bonferroni post hoc test for the main effects (standard deviation of center of pres- sure displacement in the ML, velocity in the $\mathrm{AP}$ and ML, total phase plane portrait, and ellipse area).

Using both high-top shoes and combining high-top shoes with TWO reduced the amount of displacement and velocity of the center of pressure in both AP and ML directions (Table 2). Among these parameters, standard deviation of center of pressure displacement in the ML $(\mathrm{P}<0.001)$ and velocity of displacement in both $\mathrm{AP}(\mathrm{P}=0.03)$ and $\mathrm{ML}(\mathrm{P}=0.04)$ directions were significantly decreased. The phase plane was decreased in both interventions for both the ML and AP directions, while it was only significant in the total phase $(\mathrm{P}=0.03)$ (Table 3). The path length parameter was failed to decrease significantly, but the ellipse area showed a significant effect $(\mathrm{P}<0.001)$ of interventions on the center of pressure sway (Table 3). The pairwise comparison revealed that wearing TWO along with high-top shoes significantly reduced the standard deviation in the ML direction $(\mathrm{P}<0.001)$ (Table 4). The high-top shoes were significantly effective in the standard deviation of the velocity towards AP direction $(\mathrm{P}=0.02)$. Moreover, a significant difference was found using the combined orthosis and the shoe without TWO in velocity parameters $(\mathrm{P}=0.03$ and 0.01 for $\mathrm{AP}$ and $\mathrm{ML}$, respectively) (Table 4). Both interventions were significantly improved standing balance by decreasing the ellipse area $(\mathrm{P}<0.001)$ (Table 4).

\section{Discussion}

Balance control is a crucial outcome for activities controlled by the neuromuscular systems and maintained by proprioceptive, vestibular, and visual feedback. Deficits in balance control are a major limitation in children with CP [16]. Therefore, the present study aimed to investigate the effect of using two types of conservative treatments on the balance parameters of patients with spastic diplegic CP with in-toeing. Based on the results of the present study, high-top shoes decreased the center of 
Table 1: Formulae used to calculate the center of pressure parameters.

Definition(unit)

SD of displacement in AP Direction ( $\mathrm{mm}$ )

SD of Velocity in AP Direction ( $\mathrm{mm} / \mathrm{s})$
Direction Symbol

\section{Formula}

$$
\sigma_{x}=\sqrt{\frac{\sum\left(x_{i}-\bar{x}\right)^{2}}{N-1}}
$$

$$
\sigma_{v x}=\sqrt{\frac{\sum\left(v_{x_{i}}-\bar{v}\right)^{2}}{N-1}},
$$

$$
v_{x_{i}}=\frac{\boldsymbol{x}_{(i+1)}-\boldsymbol{x}_{\boldsymbol{i}}}{\boldsymbol{t}_{(i+1)}-\boldsymbol{t}_{\boldsymbol{i}}}
$$

Phase plane portrait (arbitrary unit) in AP

AP

$\sigma_{r x}$

$\sigma_{r x}=\sqrt{\sigma_{x}^{2}+\sigma_{v_{x}}^{2}}$

SD of displacement in ML Direction (mm)

$\mathrm{ML}$

$\sigma_{y}$

$$
\sigma_{y}=\sqrt{\frac{\sum\left(y_{i}-\bar{y}\right)^{2}}{N-1}}
$$

$$
\begin{gathered}
\sigma_{v y}=\sqrt{\frac{\sum\left(v_{y_{i}}-\bar{v}\right)^{2}}{N-1}}, \\
v_{y_{i}}=\frac{\boldsymbol{y}_{(i+1)}-\boldsymbol{y}_{\boldsymbol{i}}}{\boldsymbol{t}_{(i+1)}-\boldsymbol{t}_{\boldsymbol{i}}}
\end{gathered}
$$

SD of Velocity in ML Direction ( $\mathrm{mm} / \mathrm{s})$

$\mathrm{ML}$

Phase plane portrait (arbitrary unit) in ML

$\mathrm{ML}$

$\sigma_{r y}$

$\sigma_{r y}=\sqrt{\sigma_{y}^{2}+\sigma_{v_{y}}^{2}}$

Mean Total Velocity $(\mathrm{mm} / \mathrm{s})$

Total

$\bar{V}$

$$
\bar{V}=\frac{1}{T} \sum_{t=1}^{T} \sqrt{\left(x_{i+1}-x_{i}\right)^{2}+\left(y_{i+1}-y_{i}\right)^{2}}
$$

Path Length(mm)

Total

$$
P L \quad P L=\sum_{t=1}^{T} \sqrt{\left(x_{i+1}-x_{i}\right)^{2}+\left(y_{i+1}-y_{i}\right)^{2}}
$$

Phase plane portrait (arbitrary unit) in both ML and AP (total phase)

AP-ML

$$
\sigma_{r} \quad \sigma_{r}=\sqrt{\sigma_{r_{x}}^{2}+\sigma_{r_{y}}^{2}}
$$

$$
A=2 \pi F_{0.05[2, N-2]} \sqrt{\sigma_{x}^{2} \sigma_{y}^{2}-\sigma_{x y}^{2}} \text {, }
$$

Ellipse Area

Total

A

$$
\sigma_{x y}=\sum \frac{\left(x_{i}-\bar{x}\right)\left(y_{i}-\bar{y}\right)}{N}
$$

SD: Standard deviation, AP: Anteroposterior direction, ML: Mediolateral direction, $\sigma_{x}$ : SD of displacement in AP, $\sigma_{v}:$ SD of Velocity in AP, $\sigma_{r x}$ : phase plane portrait (arbitrary unit) in AP, $\sigma_{y}$ : SD of displacement in ML, $\sigma_{v y}:$ SD of Velocity in ML, $\sigma_{r y}:$ phase plane portrait (arbitrary unit) in ML, $\bar{V}$ : Velocity, PL: Path length, $\sigma_{r}$ : Total phase, A: Ellipse area 
Table 2: Mean (SD) of aforementioned parameters in the three test conditions.

\begin{tabular}{cccccc}
\multirow{2}{*}{ Conditions } & $\boldsymbol{\sigma}_{\boldsymbol{x}}$ & $\boldsymbol{\sigma}_{\boldsymbol{y}}$ & $\boldsymbol{\sigma}_{v \boldsymbol{x}}$ & $\boldsymbol{\sigma}_{v y}$ & $\bar{V}$ \\
\cline { 2 - 5 } & Mean (SD) & Mean (SD) & Mean (SD) & Mean (SD) & Mean (SD) \\
\hline Barefoot & $9.6(4.2)$ & $6.47(2.2)$ & $42.1(10.1)$ & $31.1(9.5)$ & $45.8(13.2)$ \\
\hline Shoe & $6.2(3.7)$ & $5.5(1.1)$ & $40.7(8.7)$ & $28.5(7.3)$ & $43.6(9.5)$ \\
\hline Twister + shoe & $5.63(2)$ & $4.3(1)$ & $37.4(6.7)$ & $28.8(6.4)$ & $41.9(7.2)$ \\
\hline P-value & 0.18 & ${ }^{*} \mathrm{P}<0.001$ & ${ }^{*} \mathrm{P}=0.03$ & ${ }^{*} \mathrm{P}=0.04$ & $\mathrm{P}=0.09$ \\
\hline Effect size $\left(\eta^{2}\right)$ & 0.03 & 0.70 & 0.31 & 0.41 & 0.10
\end{tabular}

SD: Standard deviation, $\sigma_{x}$ : SD of displacement in AP (Anteroposterior direction), $\sigma_{y}$ : SD of displacement in ML (Mediolateral direction), $\sigma_{v x}$ : SD of Velocity in AP, $\sigma_{v y}$ : SD of Velocity in ML, $\bar{V}:$ Velocity, Practical/clinical meaningfulness was estimated using the partial eta squared effect size $\left(\eta^{2}\right)$ from ANOVA, where values $\geq 0.01, \geq 0.06$, and $\geq 0.14$ indicated small, medium, and large effect sizes, respectively.

*: Significant effects $(\mathrm{P}<0.05)$

Table 3: Mean (SD) of aforementioned parameters in the three test conditions

\begin{tabular}{cccccc}
\multirow{2}{*}{ Conditions } & $\boldsymbol{\sigma}_{\boldsymbol{r x}}$ & $\boldsymbol{\sigma}_{r y}$ & $\boldsymbol{\sigma}_{\boldsymbol{r}}$ & $\boldsymbol{P L}$ & $\mathbf{A}$ \\
\cline { 2 - 6 } & Mean (SD) & Mean (SD) & Mean (SD) & Mean (SD) & Mean (SD) \\
\hline Barefoot & $43.4(13.1)$ & $31.8(14.2)$ & $54.4(8.3)$ & $228.8(72.4)$ & $905.5(156.2)$ \\
\hline Shoe & $41.2(8.7)$ & $28.7(7.4)$ & $50.1(10.8)$ & $216.2(47.3)$ & $363.7(105.4)$ \\
\hline Twistert shoe & $37.9(6.9)$ & $29.01(6.4)$ & $47.4(8.35)$ & $209.3(36.6)$ & $329.4(123.7)$ \\
\hline P-value & $\mathrm{P}=0.29$ & $\mathrm{P}=0.18$ & ${ }^{*} \mathrm{P}=0.03$ & $\mathrm{P}=0.21$ & ${ }^{*} \mathrm{P}<0.001$ \\
\hline Effect size $\left(\mathrm{n}^{2}\right)$ & 0.03 & 0.10 & 0.33 & 0.08 & 0.84
\end{tabular}

SD: Standard deviation, $\sigma_{r x}$ : phase plane portrait (arbitrary unit) in AP (Anteroposterior direction), $\sigma_{r y}$ : phase plane portrait (arbitrary unit) in ML (Mediolateral direction), $\sigma_{r}$ : Total phase, PL: Path length, A: Ellipse area. Practical/clinical meaningfulness was estimated using the partial eta squared effect size $\left(\eta^{2}\right)$ from ANOVA, where values $\geq 0.01, \geq 0.06$, and $\geq 0.14$ indicated small, medium, and large effect sizes, respectively.

*: Significant effects $(\mathrm{P}<0.05)$

pressure sway in the AP direction, and wearing a high-top shoe with a strong counter could increase patients' balance parameters just in the AP direction compared to the barefoot condition. Moreover, the combine TWO and high-top shoes could significantly reduce the center of pressure sway in both ML and AP directions for children with spastic diplegic $\mathrm{CP}$, resulting in improving balance. High-top shoes alone also caused balance improvement through supporting ankle [17,28].

Children with spastic CP commonly have poor ankle control and initial contact with the forefoot during walking [27]. The present study results showed that wearing a high-top shoe alone with a strong counter could improve patients' balance parameters just in the AP direction compared the bare-foot condition. This confirmed the findings of previous studies that indicated high-top shoes resulted in balance improvement through ankle support [17, 28].

Although children with $\mathrm{CP}$ often suffer the internal rotation of the hip caused by in-toeing gait [29], the most common of this problem is a failure of physiologic correction of femoral torsion. When increased femoral anteversion is proved with some problems, the correction is recommended by a femoral derotation through orthoses [30]. According to these find- 
Table 4: Comparison of aforementioned parameters between two conditions.

\begin{tabular}{|c|c|c|c|c|}
\hline Variable & Statistical characteristic & Barefoot Vs Shoe & $\begin{array}{c}\text { Barefoot Vs } \\
\text { Twister+shoe }\end{array}$ & $\begin{array}{c}\text { Shoe Vs } \\
\text { Twister+shoe }\end{array}$ \\
\hline \multirow[b]{2}{*}{$\sigma_{y}$} & Mean Difference (SD) & $1.5(0.21)$ & $3.2(0.4)$ & $1.7(0.11)$ \\
\hline & P-value & $P=0.21$ & ${ }^{*} P<0.001$ & ${ }^{*} P<0.001$ \\
\hline \multirow{2}{*}{$\sigma_{v x}$} & Mean Difference (SD) & $3.9(0.51)$ & $4.2(0.33)$ & $0.3(0.06)$ \\
\hline & P-value & ${ }^{*} P=0.02$ & ${ }^{*} P=0.03$ & $P=0.48$ \\
\hline \multirow{2}{*}{$\sigma_{v y}$} & Mean Difference (SD) & $1.6(0.12)$ & $2.3(0.24)$ & $0.7(0.11)$ \\
\hline & P-value & $P=0.11$ & ${ }^{*} P=0.01$ & $P=0.39$ \\
\hline \multirow{2}{*}{$\sigma_{r}$} & Mean Difference (SD) & $3.5(0.47)$ & $5.2(0.6)$ & $1.7(0.21)$ \\
\hline & P-value & $P=0.41$ & ${ }^{*} P=0.03$ & $P=0.54$ \\
\hline \multirow{2}{*}{ A } & Mean Difference (SD) & $302(41)$ & $576(59)$ & $274(44)$ \\
\hline & P-value & ${ }^{*} \mathrm{P}<0.001$ & ${ }^{*} \mathrm{P}<0.001$ & $P=0.14$ \\
\hline
\end{tabular}

$\mathrm{SD}$ : Standard deviation, $\sigma_{y}: \mathrm{SD}$ of displacement in ML (Mediolateral direction), $\sigma_{v x}: \mathrm{SD}$ of Velocity in AP (Anteroposterior direction), $\sigma_{v y}:$ SD of Velocity in ML, $\sigma_{r}$ : Total phase, A: Ellipse area, VS: Versus

*: Significant effects $(\mathrm{P}<0.05)$

ings, adding a TWO to high-top shoes significantly decreased both AP and ML directions of center of pressure velocity with improving the patient's in-toeing. However, comparing the condition of high-top shoes with the hightop shoes along with TWO showed a significant difference in COP displacement in ML direction. The present study confirmed Eid et al study results who investigated the effectiveness of TWO on balance control and demonstrated TWO induced a transverse plane correction of misalignment of the lower limb [13] that is in agreement with the results of Westberry et al. study, i.e. the use of TWO could lead to control hip internal rotation [31]. Morris reported that unlike the TWO, twister cables often lead to walking more slowly in $\mathrm{CP}$ children because those increase stiffness in the lower limb, thereby making them harder for the child to walk [32]. The results of another study showed the positive effect of TWO in spatiotemporal gait parameters, whereas the twister cable was not effective on these parameters [14]. Moreover, it has been anticipated that TWO could reduce the falling rate, which has a relationship with a lack of balance in the ML direction in patients with spastic diplegic
CP [33]. An investigation reported increased balance and improved alignment of the lower limbs resulting from the lower limbs rotation to the outside and the increased feet contact with the ground using TWO [21]. Since patients' balance and base of support usually can have an improvment after increasing foot contact, the risk of falling can be also reduced [34].

Hence, prescribing TWO for patients with spastic diplegic CP, especially those who suffer from the lack of standing balance, is reasonable and suggested. As mentioned previously, the reason for the positive effect of TWO is probably due to the alignment correction between the lower limb segments while moving or standing [14]. The muscles reached their normal position with achieving the appropriate stretch and reducing balance-inappropriate moment [30].

Thus, using TWO with high-top shoes can significantly improve the position of the balance parameters in the ML direction of the center of pressure and be recommended for children with spastic diplegic CP. To the best of our knowledge, this was the first study regarding the effect of TWO attached to the 
high-top shoes on balance parameters and the findings confirmed that combined use of TWO and high-top shoe is more effective in improving balance compared with the high top shoe alone.

TWO provides gentle correctional forces to the lower limb and position muscles in the optimal length and better align the lower limb [14]. However, further research with a longterm follow-up is needed to evaluate the effect of TWO on lower limb muscles behavior. Linear analysis is a statistical summary of data, while nonlinear analyses identify the underlying structural and dynamic properties of body stability. Further, linear analysis can provide a satisfactory answer to the question of this research concerning the effect of adding TWO to the high-top shoes. More advanced analysis such as nonlinear analysis of the center of pressure in the future studies might provide better insight into the regularity of the dynamics of the center of pressure displacement during postural tasks.

Lack of follow-up period as well as control group, assessment of only one type of CP, and non-randomized test conditions were some of the limitations of this study. Therefore, further studies are suggested to consider these limitations.

\section{Conclusion}

High-top shoes alone and high-top shoes with TWO conditions might improve the balance of children with spastic diplegia more than barefoot walking. High-top shoes alone could improve postural balance in the AP direction of the center of pressure and high-top shoes with TWO improve the lower limb alignment and reduce the center of pressure sway in both ML and AP directions, i.e. combining high-top shoes with TWO may have more positive effects on improving balance parameters in children with spastic diplegic CP.

\section{Acknowledgment}

The authors would like to appreciate Dr. Aliyeh
Daryabor for editing this article.

\section{Authors' Contribution}

Rezaei M and Davoudi M. conceived of the presented idea. Ghaznavi A reffered all included patients. Shokouhyan SM and Rezaei M developed the theory and performed the computations. Davoudi M and Shokouhyan SM and Ranjbar H verified the analytical methods. All authors discussed the results and Khosravi Farsani $\mathrm{M}$ and Babaee T and Davoudi $M$ contributed to the final manuscript. Eventually all the authors read the final manuscript and approved.

\section{Ethical Approval}

The Ethics Committee also approved the study protocol (IR.SBMU.RETECH.REC.1399.372).

\section{Informed consent}

The purpose and study procedure were explained to the parents of all included children, and a written informed consent form was signed before conducting test.

\section{Funding}

Shahid Beheshti University of Medical Sciences has financially supported this study (Number: 22348).

\section{Conflict of Interest}

None

\section{References}

1. Gulati S, Sondhi V. Cerebral palsy: an overview. Indian J Pediatr. 2018;85(11):1006-16. doi: 10.1007/ s12098-017-2475-1. PubMed PMID: 29152685.

2. Cabrera MN, Snow S, Smith BP, Koman LA, Shilt JS. Acute effects of intrathecal baclofen on spinal deformity in patients with cerebral palsy. Developmental Medicine and Child Neurology. 2004;46:7.

3. Krishna KR, Priya PR, Rupanagudi A, Sankeesa S. Effect of Modified Ankle Foot Orthoses (AFO) along with Goal-Oriented Physiotherapy in a Child with Spastic Diplegic Cerebral Palsy-An Interesting Case Report. Indian Journal of Public Health Research and Development. 2021;12(2):168. doi: 10.37506/ijphrd.v12i2.14112.

4. Dodd K, Imms C, Taylor NF. Physiotherapy and occupational therapy for people with cerebral palsy: A problem-based approach to assessment and man- 
agement. John Wiley \& Sons; 2010.

5. Sankar C, Mundkur N. Cerebral palsy-definition, classification, etiology and early diagnosis. Indian J Pediatr. 2005;72(10):865-8. doi: 10.1007/ BF02731117. PubMed PMID: 16272660.

6. Dursun E, Dursun N, Alican D. Ankle-foot orthoses: effect on gait in children with cerebral palsy. Disabil Rehabil. 2002;24(7):345-7. doi: 10.1080/0963820110090724. PubMed PMID: 12022783.

7. Woollacott MH, Shumway-Cook A. Postural dysfunction during standing and walking in children with cerebral palsy: what are the underlying problems and what new therapies might improve balance? Neural Plast. 2005;12(2-3):211-9. doi: 10.1155/NP.2005.211. PubMed PMID: 16097489. PubMed PMCID: PMC2565447.

8. Binder $H$, Eng GD. Rehabilitation management of children with spastic diplegic cerebral palsy. Arch Phys Med Rehabil. 1989;70(6):482-9. doi: 10.1016/0003-9993(89)90012-9. PubMed PMID: 2658915 .

9. Mazzone S, Serafini A, Iosa M, Aliberti MN, Gobbetti T, Paolucci S, Morelli D. Functional taping applied to upper limb of children with hemiplegic cerebral palsy: a pilot study. Neuropediatrics. 2011;42(6):249-53. doi: 10.1055/s-0031-1295478. PubMed PMID: 22161724.

10. Meyns P, Kerkum YL, Brehm MA, Becher JG, Buizer Al, Harlaar J. Ankle foot orthoses in cerebral palsy: Effects of ankle stiffness on trunk kinematics, gait stability and energy cost of walking. Eur J Paediatr Neurol. 2020;26:68-74. doi: 10.1016/j. ejpn.2020.02.009. PubMed PMID: 32147412.

11. Kumar A. Orthoses in Conservative Management of Cerebral Palsy and Rehabilitation. Intech Open; 2021. doi: 10.5772/intechopen.97941. Available from: https://www.intechopen.com/chapters/76777.

12. Morris C. A review of the efficacy of lowerlimb orthoses used for cerebral palsy. Dev Med Child Neurol. 2002;44(3):205-11. doi: 10.1017/ s0012162201001943. PubMed PMID: 12005323.

13. Eid MA, Aly SM, Mohamed RA. Effect of twister wrap orthosis on foot pressure distribution and balance in diplegic cerebral palsy. J Musculoskelet Neuronal Interact. 2018;18(4):543-50. PubMed PMID: 30511958. PubMed PMCID: PMC6313041.

14. Mohamed RA, Eid MA. Twister Wraps Versus Twister Cables On Gait Pattern And In-Toeing In Children With Diplegic Cerebral Palsy. Scientific Journal of Azhar Medical Faculty (Girls).
2013;1(1):1-14.

15. Richards A, Morcos S, Rethlefsen S, Ryan D. The use of TheraTogs versus twister cables in the treatment of in-toeing during gait in a child with spina bifida. Pediatr Phys Ther. 2012;24(4):321-6. doi: 10.1097/PEP.0b013e318268a9c7. PubMed PMID: 22965202.

16. Rha DW, Kim DJ, Park ES. Effect of hinged ankle-foot orthoses on standing balance control in children with bilateral spastic cerebral palsy. Yonsei Med J. 2010;51(5):746-52. doi: 10.3349/ ymj.2010.51.5.746. PubMed PMID: 20635451. PubMed PMCID: PMC2908864.

17. Cobeljic G, Bumbasirevic M, Lesic A, Bajin Z. The management of spastic equinus in cerebral palsy. Orthopaedics and Trauma. 2009;23(3):201-9. doi: 10.1016/j.mporth.2009.05.003.

18. Kirkwood RN, Franco RD, Furtado SC, Barela AM, Deluzio KJ, Mancini MC. Frontal Plane motion of the pelvis and hip during gait stance discriminates children with diplegia levels I and II of the GMFCS. ISRN Pediatr. 2012;2012:163039. doi: 10.5402/2012/163039. PubMed PMID: 22792478. PubMed PMCID: PMC3389695.

19. Mol EM, Monbaliu E, Ven M, Vergote M, Prinzie P. The use of night orthoses in cerebral palsy treatment: sleep disturbance in children and parental burden or not? Res Dev Disabil. 2012;33(2):3419. doi: 10.1016/j.ridd.2011.10.026. PubMed PMID: 22119679.

20. Maas JC, Dallmeijer AJ, Huijing PA, BrunstromHernandez JE, et al. Splint: the efficacy of orthotic management in rest to prevent equinus in children with cerebral palsy, a randomised controlled trial. BMC Pediatr. 2012;12:38. doi: 10.1186/14712431-12-38. PubMed PMID: 22448907. PubMed PMCID: PMC3328267.

21. Chang WD, Chang NJ, Lin HY, Lai PT. Changes of plantar pressure and gait parameters in children with mild cerebral palsy who used a customized external strap orthosis: a crossover study. Biomed Res Int. 2015;2015:813942. doi: 10.1155/2015/813942. PubMed PMID: 22448907. PubMed PMCID: PMC3328267.

22. Lai YC, Lin HS, Pan HF, Chang WN, Hsu CJ, Renn $\mathrm{JH}$. Impact of foot progression angle on the distribution of plantar pressure in normal childre. Clin Biomech (Bristol, Avon). 2014;29(2):196-200. doi: 10.1016/j.clinbiomech.2013.11.012. PubMed PMID: 24342455.

23. Mazaheri M, Salavati M, Negahban $H$, Sanjari MA, Parnianpour M. Postural sway in low back pain: effects of dual tasks. Gait Posture. 2010;31(1):116- 
21. doi: 10.1016/j.gaitpost.2009.09.009. PubMed PMID: 19853454.

24. Abd El-Kafy EM. The clinical impact of orthotic correction of lower limb rotational deformities in children with cerebral palsy: a randomized controlled trial. Clin Rehabil. 2014;28(10):1004-14. doi: 10.1177/0269215514533710. PubMed PMID: 24837141.

25. Bahramizadeh M, Mousavi ME, Rassafiani M, Aminian $G$, et al. The effect of floor reaction ankle foot orthosis on postural control in children with spastic cerebral palsy. Prosthet Orthot Int. 2012;36(1):716. doi: $10.1177 / 0309364611429855$. PubMed PMID: 22130910.

26. Ferdjallah M, Harris GF, Smith P, Wertsch JJ. Analysis of postural control synergies during quiet standing in healthy children and children with cerebral palsy. Clin Biomech (Bristol, Avon). 2002;17(3):203-10. doi: 10.1016/s02680033(01)00121-8. PubMed PMID: 11937258.

27. Bahar-Özdemir Y, Ünal-Ulutatar Ç, Karali-Bingül D, Karadağ-Saygı E. Efficacy of foot-ankle orthosis on balance for children with hemiplegic cerebral palsy: An observational study. Turk J Phys Med Rehabil. 2021;67(3):336-43. doi: 10.5606/tttrd.2021.5175. PubMed PMID: 17057458.

28. Wesdock KA, Edge AM. Effects of wedged shoes and ankle-foot orthoses on standing balance and knee extension in children with cerebral palsy who crouch. Pediatr Phys Ther. 2003;15(4):22131. doi: 10.1097/01.PEP.0000096383.80789.A4. PubMed PMID: 17057458.

29. Behera P, Gopinathan NR. Examination of Gait in a Child, in Clinical Orthopedic Examination of a Child (1st ed). CRC Press; 2021. p. 18-29.

30. Hogan L. Pediatric splinting: selection, fabrication, and clinical application of upper extremity splints. Therapy Skill Builders; 1998. p. 137-51.

31. Westberry DE, Davids JR, Shaver JC, Tanner SL, Blackhurst DW, Davis RB. Impact of anklefoot orthoses on static foot alignment in children with cerebral palsy. J Bone Joint Surg Am. 2007;89(4):806-13. doi: 10.2106/JBJS.F.00694. PubMed PMID: 17403804.

32. Morris C. Orthotic management of children with cerebral palsy. J JPO. 2002;14(4):150-8. doi: 10.1016/j.mporth.2009.05.003.

33. Lawlor DA, Patel R, Ebrahim S. Association between falls in elderly women and chronic diseases and drug use: cross sectional study. BMJ. 2003;327(7417):712-7. doi: 10.1136/ bmj.327.7417.712. PubMed PMID: 14512478. PubMed PMCID: PMC200802.

34. Kadhim M, Miller F. Crouch gait changes after planovalgus foot deformity correction in ambulatory children with cerebral palsy. Gait Posture. 2014;39(2):793-8. doi: 10.1016/j.gaitpost.2013.10.020. PubMed PMID: 24316233. 